\title{
Working group report: Low energy and flavour physics
}

\author{
Coordinators: AMOL DIGHE ${ }^{1}$ and ANIRBAN KUNDU ${ }^{2}$ \\ Participants: K Agashe ${ }^{3}$, B Anantanarayan ${ }^{4}$, A Chandra ${ }^{1}$, A Datta ${ }^{5}$, P K Das ${ }^{6}$, \\ S P Das ${ }^{5}$, A Dighe ${ }^{1}$, R Forty ${ }^{7}$, D K Ghosh ${ }^{8}$, Y -Y Keum ${ }^{9}$, A Kundu ${ }^{2}$, N Mahajan ${ }^{6}$, \\ S Majhi ${ }^{6}$, G Mazumdar ${ }^{1}$, K Mazumdar ${ }^{1}$, P Mehta ${ }^{6,10}$, Y Nir ${ }^{11}$, J P Saha ${ }^{5}$, R Singh ${ }^{4}$, \\ N Sinha ${ }^{12}$, R Sinha ${ }^{12}$, A Soni $^{13}$, S Uma Sankar ${ }^{14}$ and R Vaidya ${ }^{15}$ \\ ${ }^{1}$ Tata Institute of Fundamental Research, Homi Bhabha Road, Mumbai 400 005, India \\ ${ }^{2}$ Department of Physics, University of Calcutta, 92 A. P. C. Road, Kolkata 700 009, India \\ ${ }^{3}$ Department of Physics, Johns Hopkins University, Baltimore, MD 21218, USA \\ ${ }^{4}$ Centre for High Energy Physics, Indian Institute of Science, Bangalore 560 012, India \\ ${ }^{5}$ Department of Physics, Jadavpur University, Kolkata 700 032, India \\ ${ }^{6}$ Harish-Chandra Research Institute, Chhatnag Road, Jhusi, Allahabad 211 019, India \\ ${ }^{7}$ CERN, CH-1211 Geneva 23, Switzerland \\ ${ }^{8}$ Institute of Theoretical Science, University of Oregon, Eugene, OR 97403, USA \\ ${ }^{9}$ Department of Physics, Nagoya University, Nagoya, 464-6801 Japan \\ ${ }^{10}$ Department of Physics and Astrophysics, University of Delhi, Delhi 110 019, India \\ ${ }^{11}$ Department of Particle Physics, Weizmann Institute of Science, Rehovot 76100, Israel \\ ${ }^{12}$ Institute of Mathematical Sciences, CIT Campus, Taramani, Chennai 600 113, India \\ ${ }^{13}$ Brookhaven National Laboratory, Upton, NY 11973, USA \\ ${ }^{14}$ Department of Physics, Indian Institute of Technology, Mumbai 400 076, India \\ ${ }^{15}$ Department of Physics, National Central University, Chung-Li 32054, Taiwan \\ E-mail: amol@theory.tifr.res.in
}

Abstract. This is a report of the low energy and flavour physics working group at WHEPP-8, held at the Indian Institute of Technology, Mumbai, India, during 5-16 January 2004.

Keywords. $B$ physics, perturbative quantum chromodynamics.

PACS No. 13.25.Hw

\section{Introduction}

The activities of the 'low energy and flavour physics' working group at WHEPP-8 were almost exclusively in the area of $B$ physics. Given that data from the $B$ factories are currently challenging the predictions of theoretical models as well as 
offering teasing hints of physics beyond the standard model, this should not be surprising.

There were four plenary talks, starting with 'CP violation: a new era' by $\mathrm{Y}$ Nir, which presented the current status of our understanding of charge-parity violation in the quark sector. This was followed by ' $B$ s physics at LHCb' by R Forty and 'Improved searches for new physics at a super- $B$ factory' by A Soni, which discussed future experiments focussed on the study of $B$ mesons and pointed out the decay modes that have the potential of revealing new physics. Y-Y Keum, in his talk on 'Charmless hadronic decays of $B$ mesons', described the pQCD approach to calculate the hadronic decay rates more reliably. All these talks are published in these proceedings.

In addition, there were five working group talks:

- Nita Sinha: $B \rightarrow V_{1} V_{2}$ decays and new physics,

- Kaustubh Agashe: Flavour physics from Randall-Sundrum model,

- Amarjit Soni: $\varepsilon^{\prime} / \varepsilon$ on and off the lattice,

- Yong-Yeon Keum: Rare $B$ decays from perturbative QCD,

- Rishikesh Vaidya: Radiative $B$ decays from $R$-parity violating supersymmetry.

A special mention must be made of the series of informal lectures by Y-Y Keum on using the techniques of perturbative QCD to compute the $B$ meson decay amplitudes. With the $B$ factories coming up with more and more accurate measurements of the decay rates of $B$ mesons to various final states, the naive factorization is no longer sufficient to explain all the data and QCD corrections have to be taken into account. Y-Y Keum went painstakingly over the nittygritties of the pQCD approach that calculates the non-leptonic decay amplitudes including the long-distance contributions. There were three lectures that lasted for over seven hours, and were attended by a number of participants.

A brief review of the problems discussed during the workshop follows.

\title{
2. Model-independent study of $B \rightarrow \eta K$ modes
}

\author{
A Dighe, A Kundu, J P Saha, A Soni and S Uma Sankar
}

The mixing-induced $\mathrm{CP}$ asymmetry $A_{\mathrm{CP}}^{\text {mix }}$ in $B_{d} \rightarrow J / \psi K_{S}$ mode is $\sin (2 \beta)$, which has been measured with a precision of about $5 \%$. The two experiments BaBar and Belle are in perfect agreement, and the results from all charmonium modes are consistent with each other. This is no surprise, since this decay is dominated by the $b \rightarrow c \bar{c} s$ tree amplitude, and the penguin amplitude that comes with a different weak phase makes a negligible contribution.

The asymmetry $A_{\mathrm{CP}}^{\mathrm{mix}}$ measured in the mode $B_{d} \rightarrow \phi K_{S}$ should also be $\sin (2 \beta)$ in SM. However, the current average is almost $3 \sigma$ away from the $B_{d} \rightarrow J / \psi K_{S}$ value [1]. If this discrepancy is indeed real, it cannot be accounted for just by uncertainties in the theoretical models. All the models (naive factorization, QCD factorization, 
perturbative QCD) predict the penguin $b \rightarrow s \bar{s} s$ contribution to be overwhelmingly dominant over the tree one, and therefore predict the mixing-induced CP asymmetry to be equal to $\sin (2 \beta)$.

The measurements of this quantity in BaBar and Belle have large errors and still are almost incompatible. So it is premature to conclude that the above is any evidence for physics beyond SM. However, if the anomaly persists, it would become important to check if it is indeed due to some new physics, or due to our lack of understanding of the penguin dynamics.

It is the relative magnitudes of tree and penguin amplitudes in the decay that determine how different $A_{\mathrm{CP}}^{\mathrm{mix}}$ can be from $\sin (2 \beta)$. Hence it is worthwhile to look at the ratio of these magnitudes in related decays. In this context, we consider the decay channels $B^{0}\left(B^{+}\right) \rightarrow \eta^{\left({ }^{\prime}\right)} K^{0(*)}\left(K^{+(*)}\right)$, and specifically the decay $B^{0} \rightarrow \eta^{\prime} K^{0}$. The amplitude of this decay can be written in general as the sum of a 'tree' and a 'penguin' amplitude. A 'tree' amplitude here is just a term that is associated with the weak phase $\operatorname{Arg}\left(V_{u b}^{*} V_{u s}\right)=-\gamma$ and a 'penguin' amplitude is the one which comes with the weak phase $\operatorname{Arg}\left(V_{t b}^{*} V_{t s}\right)=\pi$. The net amplitude is

$$
A\left(\bar{B}^{0} \rightarrow \eta^{\prime} \bar{K}^{0}\right)=T \mathrm{e}^{-i \gamma} \mathrm{e}^{i \delta_{T}}+P \mathrm{e}^{i \delta_{P}} \mathrm{e}^{i \pi}=P \mathrm{e}^{i \delta_{P}}\left(-1+r \mathrm{e}^{-i \gamma} \mathrm{e}^{-i \delta}\right),
$$

where $r \equiv|T / P|$, and the strong phase difference is $\delta \equiv \delta_{P}-\delta_{T}$. The ratio $r$ is expected to be $\ll 0.1$ in almost all theoretical models. The strong phase difference $\delta$ is also expected to be small from colour transparency arguments. The CP asymmetries are given by

$$
A_{\mathrm{CP}}^{\mathrm{dir}}=\frac{1-R^{2}}{1+R^{2}}, \quad A_{\mathrm{CP}}^{\mathrm{mix}}=\frac{2 R}{1+R^{2}} \sin 2(\beta+\zeta),
$$

where $R$ and $\zeta$ are functions of $r, \gamma$ and $\delta$ through

$$
R \mathrm{e}^{-2 i \zeta} \equiv \frac{\left\langle\eta^{\prime} \bar{K}^{0} \mid \bar{B}^{0}\right\rangle}{\left\langle\eta^{\prime} K^{0} \mid B^{0}\right\rangle}
$$

The CP asymmetry data provides a correlation between the ratio of the tree and the penguin amplitudes and the weak phase $\gamma$, whereas the branching ratio gives the absolute value of the amplitudes. We show in figure 1 the constraints on the four unknowns $T, r, \gamma, \delta$ in terms of three observables, the branching ratio, $A_{\mathrm{CP}}^{\text {dir }}$, and $A_{\mathrm{CP}}^{\mathrm{mix}}$, taking into account the best-fit values [1] and $1 \sigma$ error bars. We vary $r$ between 0 and $+1, \gamma$ between 0 and $\pi$, and $\delta$ between $-\pi$ and $\pi$ with a uniform distribution. The SM value of $\sin (2 \beta)$ is taken to be the average obtained from the charmonium modes, since such a value is also independently supported from the SM fit.

It is clear that $r$ needs to be at least $\sim 0.1$, and even greater if the value of $\gamma$ is away from $\pi / 2$. Thus, the $B^{0} \rightarrow \eta^{\prime} K^{0}$ mode independently suggests that there is a significant 'tree pollution'. The measurement of $A_{\mathrm{CP}}^{\mathrm{mix}}$ also gives a lower bound on $r$. On the other hand, the direct $\mathrm{CP}$ measurement data demand that the strong phase $\delta$ be small: at larger values of $\delta$ the allowed parameter space shrinks, as can be seen from figure 1 . The interference between the tree and penguin amplitude gives rise to two solutions for $T$ for a single value of $r$, as the right-hand panel shows. However, the lower branch becomes disallowed as $\delta$ goes away from zero.

Pramana - J. Phys., Vol. 63, No. 6, December 2004 

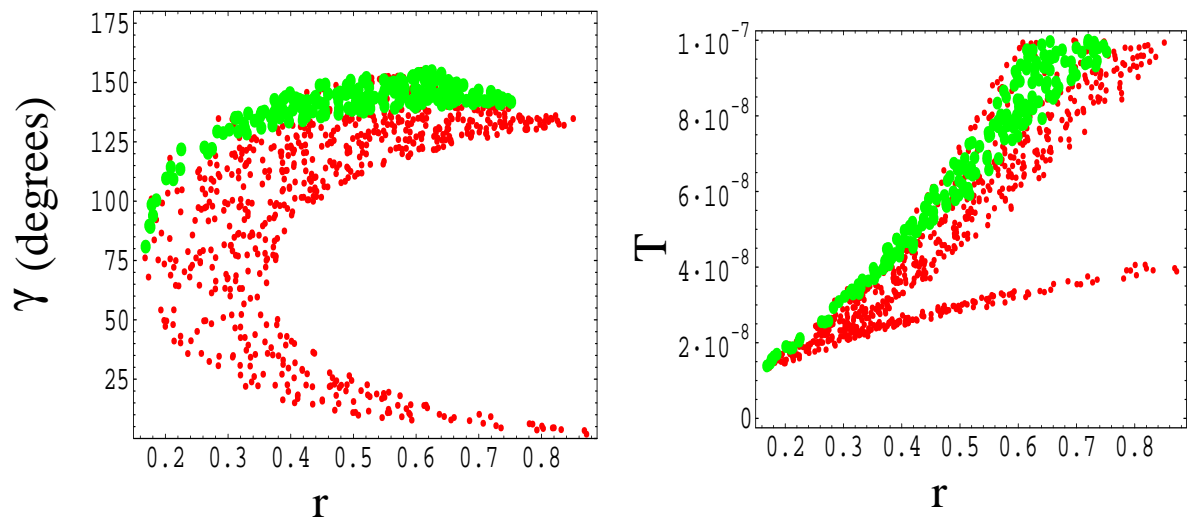

Figure 1. The values of $r, \gamma$ and $T$ allowed at $1 \sigma$ with the current data. Black dots denote the allowed values with $\delta=0$, Grey circles denote the allowed values with $\delta=\pi / 12$.

The analysis can be further improved by including the data from $B^{+} \rightarrow \eta^{\left({ }^{\prime}\right)} K^{+}$ and $B \rightarrow \eta^{\left({ }^{\prime}\right)} K^{*}$. Since the experimental errors are large at this stage, the above observations are just indicative, but with more data, strong limits can be put on the parameters $r, \gamma$ and $\delta$. This may provide a hint towards the solution of the $\phi K_{S}$ anomaly, if it persists.

\section{New physics contributions to $b \rightarrow s$ penguins}

\section{P K Das, Y-Y Keum, A Kundu, K Mazumdar, Y Nir, J P Saha, N Sinha and R Sinha}

Although the rate of the radiative inclusive decay $b \rightarrow s \gamma$ as well as its exclusive counterpart is in complete agreement with the SM predictions, measurements of $b \rightarrow s$ penguins in exclusive hadronic channels still pose problems for theoretical models. The anomaly observed in the indirect $\mathrm{CP}$ asymmetry in $B \rightarrow \phi K_{S}$ is one such example. There also seems to be an abnormally large electroweak penguin contribution in $B \rightarrow \pi K$ channels. If all these anomalies are related to the same source, viz. some new physics in the $b \rightarrow s$ penguin, then we can try to determine the nature of this new physics by studying various channels that involve this diagram.

This project initiated the process of listing all possible new physics operators for the four-Fermi decay $b \rightarrow s \bar{s} s$, and examining the correlated signals in other channels. Obviously, the signals will be different for different Lorentz structures. For example, an operator of the form $\left(\bar{s} \gamma_{\mu} \gamma_{5} s\right)\left(\bar{s} \gamma^{\mu} b\right)$ should contribute to $B \rightarrow$ $\eta^{\prime} K_{S}$ but not to $B \rightarrow \phi K_{S}$, whereas if the operator were $V \otimes V$ instead of $A \otimes V$, it is the $\phi K_{S}$ channel that will be affected. This list goes on, and one can add operators of the form $(V-A) \otimes(V+A),(V+A) \otimes(V-A)$, etc. 
The task is as follows:

- Survey all possible excitations in the final state $P P, P V$, and $V V$ channels to check which ones are affected by this new physics.

- Study the effects in the $B_{\mathrm{s}}$ system, in particular on channels like $J / \psi \phi$ and $\phi \phi$ and the angular distributions of their eventual decay products.

- Estimate how the determination of $\gamma$ is affected for channels like $B_{\mathrm{s}} \rightarrow$ $K^{+} K^{-}, B_{\mathrm{s}} \rightarrow D_{\mathrm{s}}^{+} D_{\mathrm{s}}^{-}$, etc. and look for any correlations.

\section{4. pQCD for calculating $B$ decay amplitudes}

\section{R Vaidya, R Singh, J P Saha, S Majhi, N Mahajan, Y-Y Keum, A Dighe and S P Das}

The increasing accuracy of measurements at the $B$ factories has brought new challenges for the theory trying to explain these results. It is now an accepted fact that the naive factorization approach, which seemed very successful in explaining most of the decays previously, receives large corrections. In the past couple of years, estimation of these so-called 'non-factorizable' sub-leading corrections has been one of the main focal points of intense activity. In this direction, two main approaches have been employed, QCD factorization [2] and the perturbative QCD (pQCD) approach $[3,4]$.

In the naive factorization approach, the amplitude for a typical decay process involving two mesons in the final state is assumed to be the convolution of the respective hadron wave functions with the perturbative hard decay kernel, assuming that there are no gluon exchanges between the final state hadrons. The wave functions and various form factors are the non-perturbative inputs obtained from lattice or sum rule methods. The QCD factorization method includes the nonfactorizable corrections arising due to gluonic exchanges between the final state hadrons as well, but the form factors are still treated as non-perturbative inputs and therefore universal in character. The pQCD approach, in contrast, advocates the idea that form factors can be reliably computed in perturbation theory and only the wave functions/parton distribution amplitudes are the basic non-perturbative inputs.

The amplitude in pQCD can be symbolically expressed as [5]

$$
A \sim C(t) \times H(t) \times \Phi(x) \times \exp \left[-s(P, b)-2 \int_{1 / b}^{t} \frac{\mathrm{d} \mu}{\mu} \gamma_{q}\left(\alpha_{\mathrm{s}}(\mu)\right)\right],
$$

where $t$ is the factorized scale in the hard part $H(t)$ that is perturbatively calculable, $C(t)$ are Wilson coefficients, and $\Phi(x)$ are meson light cone distribution amplitudes. The 'Sudakov form factor' $\exp [-s(P, b)]$ arises from the resummation of terms involving $\log ^{2}(b P)$ where $P$ is the dominant light cone component of the meson momentum and $1 / b$ is the transverse momentum below which the dynamics is completely non-perturbative. This takes care of the suppression of long-distance contribution near the end-point $1 / b \sim \Lambda_{\mathrm{QCD}}$. The infra-red divergence appearing 
at low momentum fraction $x$ from terms involving $\log ^{2}(1 / x)$ is taken care of by the so-called 'threshold resummation', which introduces a universal 'Sudakov factor' that is absorbed in the wave function. With these two end-point behaviours taken care of, the physical quantities calculated turn out to be finite.

The pQCD approach thus can calculate form factors of the type $B \rightarrow P, V$. It also generates the strong phases in a manner different from the other methods. The method has been applied to many channels and the results for the rates, form factors and various asymmetries in the $B \rightarrow P P$ modes are very encouraging [5]. The absolute reliability of the approach is an open question and the best way is to use this method to calculate quantities that can be measured experimentally.

During the second week of the workshop, Y-Y Keum readily agreed to give a couple of informal lectures, elaborating on various technical points and computational details. In particular, it was demonstrated how to get strong phases in this approach. Using the decay mode $B \rightarrow K \pi$ as an example, various aspects of the pQCD approach were outlined in great detail.

It was planned to investigate $B_{\mathrm{s}} \rightarrow J / \psi \phi$ and $B_{\mathrm{s}} \rightarrow \phi \phi$ modes, calculate the helicity amplitudes involved, and study the angular correlations and $\mathrm{CP}$ asymmetries. These modes are crucial for the measurement of lifetime difference $\Delta \Gamma_{\mathrm{s}}$ and the weak phase $\beta_{\mathrm{s}}$, and statistically significant amount of data from these modes is expected to be available within the next few years.

\section{5. $\Delta \Gamma / \Gamma$ and $C P T$ violating observables in neutral $B$ systems}

\section{A Datta, A Dighe, R Forty, A Kundu, G Majumdar and K Mazumdar}

In both the $B_{\mathrm{s}}-\bar{B}_{\mathrm{s}}$ as well as $B_{d}-\bar{B}_{d}$ systems, the mass eigenstates have slightly different lifetimes. In the $B_{\mathrm{s}}$ system, the theoretical estimation of $(\Delta \Gamma / \Gamma)_{\mathrm{s}}$ is roughly $0.09 \pm 0.04[6]$ whereas the experimental upper limit is 0.29 . The measurement of $(\Delta \Gamma / \Gamma)_{\mathrm{s}}$ is already being attempted at $\mathrm{CDF}$ and $\mathrm{D} \varnothing$, through the lifetimes of decays like $B_{\mathrm{s}} \rightarrow J / \psi \phi, J / \psi \eta^{\prime}$. The prospects of measuring $(\Delta \Gamma / \Gamma)_{\mathrm{s}}$ at LHC have already been studied in detail [7].

In the $B_{d}$ system, the lifetime difference should be much smaller: the theoretical estimation is $(\Delta \Gamma / \Gamma)_{d} \approx(0.3 \pm 0.1) \%$ [8], and the measurement is beyond the ability of the current experiments. The feasibility studies of measurement of $(\Delta \Gamma / \Gamma)_{d}$ at $B$-factories indicate that a limit of $(\Delta \Gamma / \Gamma)_{d}<2 \%$ may be possible at BaBar or Belle with $500 \mathrm{fb}^{-1}[9]$, but one needs super- $B$ factories for an actual measurement. The most promising method for this would be the measurement of the difference of 'effective' lifetimes of the self-tagging decays and the decays to $\mathrm{CP}$ eigenstates without direct $\mathrm{CP}$ violation. The ratio of the two lifetimes $\tau_{\mathrm{SL}}$ and $\tau_{\mathrm{CP} \pm}$ is

$$
\frac{\tau_{\mathrm{SL}}}{\tau_{\mathrm{CP} \pm}}=1 \pm \frac{\cos (2 \beta)}{2} \frac{\Delta \Gamma}{\Gamma_{d}}+\mathcal{O}\left[\left(\Delta \Gamma / \Gamma_{d}\right)^{2}\right]
$$

The measurement of these two lifetimes to an accuracy of better than $0.2 \%$ should be able to give us a measurement of $(\Delta \Gamma / \Gamma)_{d}$. In spite of the uncertainties in the theoretical calculations of the lifetime difference, the measurements are still 
desirable since they lead to observables where new physics can be detected even without having to refer to the theoretical predictions [8].

The measurement of $(\Delta \Gamma / \Gamma)_{d}$ at hadronic machines had not yet been explored. At $\mathrm{LHCb}$, the good particle ID for charged pions and kaons makes decay modes like $\pi^{+} \pi^{-}$very attractive. However, this mode involves significant direct CP violation. The feasibility study for measuring $(\Delta \Gamma / \Gamma)_{d}$ through this mode should be undertaken.

The decays and mixings of $B$ mesons can be affected by CPT violating effects. The measurements of such effects may be hampered by the presence of a significant ' $\Delta \Gamma$ ' pollution. For example, the direct $\mathrm{CP}$ asymmetry,

$$
a_{\mathrm{CP}}^{\mathrm{dir}}=\frac{\left|\left\langle f \mid B^{0}(t)\right\rangle\right|^{2}-\left|\left\langle f \mid \bar{B}^{0}(t)\right\rangle\right|^{2}}{\left|\left\langle f \mid B^{0}(t)\right\rangle\right|^{2}+\left|\left\langle f \mid \bar{B}^{0}(t)\right\rangle\right|^{2}}
$$

is time dependent in the presence of CPT violation if the lifetime difference is vanishingly small. However, time dependence may also arise in the presence of a significant $(\Delta \Gamma / \Gamma)_{d}[10]$. One can put limits on the fake CPT violation induced by the lifetime difference, and explore the feasibility of disentangling these two quantities in future experiments.

\section{References}

[1] Heavy Flavor Averaging Group homepage: http://www.slac.stanford.edu/xorg/hfag/

[2] M Beneke, G Buchalla, M Neubert and C T Sachrajda, Nucl. Phys. B606, 245 (2001)

[3] Y Y Keum, H N Li and A I Sanda, Phys. Rev. D63, 054008 (2001)

[4] Y Y Keum, H N Li and A I Sanda, Phys. Lett. B504, 6 (2001)

[5] Y Y Keum and A I Sanda, Phys. Rev. D67, 054009 (2003)

[6] M Beneke and A Lenz, J. Phys. G27, 1219 (2001)

[7] P Ball et al, $B$ decays at the LHC, hep-ph/0003238

[8] A S Dighe, T Hurth, C S Kim and T Yoshikawa, Nucl. Phys. B624, 377 (2002)

[9] M Battaglia et al, The CKM matrix and the unitarity triangle, hep-ph/0304132

[10] A Datta, E A Paschos and L P Singh, Phys. Lett. B548, 146 (2002) 